\title{
Potencialidades e limitações da atuação do enfermeiro no Centro Parto Normal
}

\author{
Potentialities and limitations of nurses' performance in the Normal Birth Center \\ Potencialidades y limitaciones del desempeño de las enfermeras en el Centro Parto Normal
}

\begin{abstract}
Antonio Rodrigues Ferreira Júnior ${ }^{1}$ (1) Luciana Camila dos Santos Brandão ${ }^{1}$ (B) Ana Carolina de Melo Farias Teixeira ${ }^{1}$ (C) Alexandrina Maria Ramos Cardoso 2 (B)

1. Universidade Estadual do Ceará. Fortaleza, CE, Brasil.
\end{abstract}

2. Escola Superior de Enfermagem do Porto. Porto, Portugal.
Autor correspondente:

Antonio Rodrigues Ferreira Júnior.

E-mail: arodrigues.junior@uece.br

Recebido em 08/03/2020.

Aprovado em 21/08/2020.

DOl:https://doi.org/10.1590/2177-9465-EAN-2020-0080

\section{RESUMO}

Objetivo: Conhecer as potencialidades e limitações da atuação do enfermeiro no Centro de Parto Normal (CPN). Método: abordagem qualitativa, do tipo exploratório e descritivo, realizado em 2018, com seis enfermeiras atuantes em CPN intrahospitalar público na região metropolitana de Fortaleza, Ceará, Brasil. A coleta das informações ocorreu por meio de entrevista individual, com análise a partir dos pressupostos da sociologia das profissões, com foco nos temas: conhecimento e autonomia; credencialismo; divisão do trabalho; mercado de trabalho e quadro de valores. Resultado: A atuação do enfermeiro no CPN potencializa as boas práticas para o parto e nascimento, bem como amplia a importância e visibilidade deste profissional no cuidado materno-infantil. O cuidado clínico e a gestão emergem como foco da ação do enfermeiro no CPN. No entanto, mesmo com a indução do Estado para essa atuação, ainda há a necessidade de reconhecimento das competências e autonomia do enfermeiro no cuidado obstétrico por outros profissionais. Conclusão e implicações para a prática: Há desafios que precisam ser superados como a ampliação da autonomia e do respeito ao credenciamento do enfermeiro para atuação no CPN e a harmonização entre a gestão do processo de trabalho e gestão do cuidado clínico por este profissional.

Palavras-chave: Enfermeiras Obstétricas; Parto Humanizado; Papel Profissional. Autonomia Profissional; Mercado de Trabalho.

\section{ABSTRACT}

Objective: To know the potentialities and limitations of the nurse's performance in the Center for Normal Birth (CPN). Method: Qualitative approach, exploratory-descriptive type, carried out in 2018, with six nurses working in public intra-hospital CPN in the metropolitan region of Fortaleza, Ceará, Brazil. The collection of information occurred through individual interview, with analysis from the assumptions of the sociology of professions, focusing on the themes: knowledge and autonomy; credentialism; division of labor; labor market and value chart. Result: The nurse's performance in the Center for Normal Birth (CPN)enhances good practices for childbirth and birth, as well as increases the importance and visibility of this professional in maternal and child care. Clinical care and management emerge as the focus of the nurse's action in the CPN. However, even with the induction of the State to this action, there is still the need for recognition of the competencies and autonomy of the nurse in obstetric care by other professionals. Conclusion and implications for practice: There are challenges that need to be overcome such as the expansion of autonomy and respect for the accreditation of the nurse to work in the CPN and the harmonization between the management of the work process and management of clinical care by this professional.

Keywords: Obstetric Nurses; Humanized Birth; Professional Role. Professional Autonomy; Labor Market.

\section{RESUMEN}

Objetivo: Conocer las potencialidades y limitaciones del desempeño de la enfermera en el Centro de Parto Normal (CPN) Método: Enfoque cualitativo, del tipo exploratorio-descriptivo, realizado en 2018, con seis enfermeras que trabajan en el CPN intrahospitalario público en la región metropolitana de Fortaleza, Ceará, Brasil. La recopilación de información se realizó mediante una entrevista individual, con análisis basado en los supuestos de la sociología de las profesiones, centrándose en los temas: conocimiento y autonomía; credencialismo; división del trabajo; mercado de trabajo y tabla de valores. Resultado: El desempeño de la enfermera en la CPN mejora las buenas prácticas para el parto y el nacimiento, así como aumenta la importancia y la visibilidad de este profesional en el cuidado de la madre y el niño. El cuidado clínico y la gestión surgen como el foco de la acción de la enfermera en la CPN. Sin embargo, incluso con la inducción del Estado a esta acción, sigue siendo necesario que otros profesionales reconozcan las aptitudes y la autonomía de la enfermera en la atención obstétrica por otros profesionales. Conclusión e implicaciones para la práctica: Hay desafíos que deben superarse, como el aumento de la autonomía y el respeto de la acreditación de las enfermeras para trabajar en la CPN y la armonización de la gestión del proceso de trabajo y la gestión de la atención clínica por parte de este profesional.

Palabras clave: Enfermeras obstétricas; Parto humanizado; Papel profesional. Autonomía profesional; Mercado de trabajo. 


\section{INTRODUÇÃO}

O cenário da assistência à mulher no parto tem sido influenciado por mudanças que ocorrem no Brasil, ${ }^{1}$ especialmente a mobilização, tanto das parturientes quanto das instituições de saúde e profissionais, em busca de uma assistência menos intervencionista e de um nascimento humanizado.

O cuidado voltado para as necessidades da parturiente é potencializado a partir da conscientização das mulheres na luta pelos seus direitos e na reivindicação de melhorias das condições de vida. Nesse âmbito, a Organização Mundial da Saúde (OMS) e o Fundo de População das Nações Unidas recomendam a liderança e o envolvimento de uma parteira ou enfermeira com habilidades de obstetrícia no pré-natal, assim como para o manejo do parto vaginal. , $^{2,3}$

Isto ocorre porque o modelo de assistência intervencionista, apesar de justificado pelo uso da tecnologia para melhor eficiência no trabalho de parto e para a segurança da gestante e do recémnascido, está correlacionado com piores resultados maternos e neonatais no panorama nacional e internacional. Circunstância essa que despertou a mobilização dos profissionais da saúde pública, na busca por modificação dos padrões da assistência obstétrica brasileira. ${ }^{4-7}$

À vista disso, para a efetivação desse novo modelo de atenção obstétrica, ressalta-se a criação da Rede Cegonha. Trata-se de uma rede de cuidados que visa assegurar à mulher o direito ao planejamento reprodutivo e à atenção humanizada à gravidez, ao parto e ao puerpério, bem como direito ao nascimento seguro, ao crescimento e desenvolvimento da criança até os dois anos. ${ }^{8}$

Nesse âmbito, em busca de uma assistência mais humanizada à mulher, voltada para as necessidades da parturiente e para a redução da taxa de mortalidade, o Ministério da Saúde estabeleceu diretrizes para a implantação e habilitação do Centro de Parto Normal (CPN) no âmbito do Sistema Único de Saúde (SUS). ${ }^{9}$ No entanto, por ser um novo cenário de práticas no sistema de saúde, a atuação do enfermeiro ainda se encontra em um campo de construções e disputas.

A inclusão da enfermeira obstétrica no enfrentamento das adversidades apresentadas a partir da indução do Estado, com financiamento para formação na área, construção e custeio de novos equipamentos de saúde como o CPN, surge como ação importante no cenário obstétrico brasileiro. Isso possibilita o fortalecimento da profissão na cena do parto de risco habitual. ${ }^{10}$

Novas áreas da enfermagem surgem a partir das modificações construídas socialmente e, muitas vezes, impulsionadas pelo Estado, visto que possuem uma atuação protagônica no serviço público. A reorganização contínua do sistema de saúde para prover respostas às demandas sociais exige adaptação da enfermagem para as novas realidades e pode ser vista como evolução profissional importante e necessária. ${ }^{11,12}$

Destarte, os CPN contam com uma atuação autônoma das enfermeiras obstétricas em situações consideradas de risco habitual, além de serem responsáveis pela valorização do parto natural, permitindo que a mulher participe ativamente do processo. Salienta-se a relevância da atuação da enfermeira obstétrica no cenário de assistência ao parto e nascimento de risco habitual, em razão de um cuidado menos intervencionista, inerente à sua formação, bem como na promoção do uso de boas práticas baseadas em evidências e sensibilização para o protagonismo da mulher no processo de parturição. ${ }^{8-10}$

Nesse contexto, questiona-se: como ocorre a atuação do enfermeiro no CPN? Quais as práticas de cuidado e de gestão desenvolvidas pelos enfermeiros neste equipamento de saúde? A partir disso, o presente estudo objetivou conhecer as potencialidades e limitações da atuação do enfermeiro no Centro de Parto Normal.

\section{MÉTODO}

Estudo com abordagem qualitativa, do tipo exploratório e descritivo, ${ }^{13}$ realizado em 2018 , com a participação de seis enfermeiras atuantes em CPN intra-hospitalar, localizado em instituição pública da região metropolitana de Fortaleza, Ceará, escolhido por ter sido o primeiro centro habilitado pelo Ministério da Saúde no Estado. Todas as enfermeiras da equipe participaram da pesquisa, a partir do critério de inclusão: trabalhar no CPN há seis meses, no mínimo. Embora tenham sido utilizados como critérios de exclusão estar em férias ou licença no momento da coleta de informações, não houve enfermeiros nestas condições.

O equipamento de saúde se configura como importante ponto de atenção, oferecendo assistência durante partos de risco habitual aos oito municípios que compõem sua respectiva região de saúde. Realiza em média 300 partos por mês com três quartos pré-parto, parto e pós-parto e é campo para vivências práticas da graduação em enfermagem e residência em enfermagem obstétrica de universidades públicas.

A coleta das informações ocorreu entre julho e agosto de 2018 , por meio de entrevista individual em profundidade com 30 minutos de duração em média, realizada por uma das pesquisadoras em sala disponibilizada para esta finalidade, com registro em gravador de voz, a partir da autorização das participantes. A entrevista abordou, em questões abertas, a percepção dos enfermeiros sobre aspectos das práticas profissionais no CPN, incluindo a sua rotina, a atuação como parte da equipe, as ações de cuidado e de gestão do enfermeiro no CPN, o significado de cuidado para estes profissionais, as dificuldades e as facilidades para implementação das boas práticas ao parto e nascimento, bem como as mudanças necessárias para facilitar a implementação destas práticas.

A análise temática ${ }^{13}$ foi utilizada para a organização das informações, a partir da construção de um quadro com as narrativas dos participantes, extração das unidades de sentido e, posteriormente, as unidades de contexto, considerando as temáticas elencadas na sociologia das profissões, ${ }^{14}$ que norteou teoricamente a análise. Destaca-se que todos os pesquisadores participaram desta etapa. Os resultados serão apresentados divididos nos seguintes temas: Conhecimento e Autonomia; Credencialismo; Divisão do Trabalho; Mercado de Trabalho e Quadro de Valores. 
A sociologia das profissões consiste em analisar e compreender as manifestações do profissionalismo na emergência e desenvolvimento de profissões e grupos ocupacionais que são marcados pela diversidade de modelos de organização, pela heterogeneidade interna, pela imprecisão das fronteiras que os separam, pela diversidade de critérios de unificação e por outras especificidades decorrentes de contextos históricos e geopolíticos associados à sua gênese. ${ }^{14} \mathrm{Na}$ perspectiva deste estudo, é utilizada como base para discutir as práticas profissionais de enfermeiros em Centro de Parto Normal, equipamento de saúde inserido no sistema público e regulamentado pelo Ministério da Saúde no ano de 2013. ${ }^{\circ}$

Salienta-se que foi utilizado o instrumental Consolidated Criteria for Reporting Qualitative Research (COREQ), com o intuito de proporcionar transparência ao processo realizado na pesquisa. ${ }^{15}$ Ademais, o estudo foi aprovado pelo Comitê de ética da Universidade Estadual do Ceará, em 01 de agosto de 2017, com parecer no 2.195.430, seguindo a Resolução nํ466, do Conselho Nacional de Saúde. ${ }^{16}$

\section{RESULTADOS}

As participantes foram do sexo feminino, com tempo de trabalho no CPN entre um a 10 anos, faixa de idade de 26 a 59 anos, cinco das quais possuíam especialização em enfermagem obstétrica e uma concluiu a residência uniprofissional em enfermagem obstétrica.

No tema Conhecimento e autonomia foram apresentados os seguintes relatos:

Mas tem muita coisa aqui, é um campo riquíssimo, é muito prazeroso, é muito bom, a gente tem muita autonomia, mas tem a questão de ter muita responsabilidade também. (E6)

No CPN a gente tem que trabalhar com as melhores evidências científicas para prestar o melhor cuidado. O enfermeiro precisa saber o que está fazendo e isso exige muito estudo constante. Não é fácil, mas a gente escolheu a enfermagem obstétrica, então é isso. (E2)

...acho que a gente acaba fazendo bastante é isso, educar, orientar. [...]Eu acho que também a questão de tranquilizar, transmitir apoio, transmitir segurança, principalmente. Mas nem sempre isso é possivel porque é muita coisa para fazer. (E6)

Com relação ao tema Credencialismo, as enfermeiras, por sua vez, falaram:

Aqui não tem médico, aqui são só os enfermeiros e os técnicos. O médico, nós chamamos quando julgamos necessário, quando tem alguma intercorrência. Aqui somos nós que fazemos os partos. (E4)

Então, é muito importante todo mundo saber e falar a mesma coisa para não pegar uma pessoa questionadora
[...] senão ele vai achar que esse profissional não sabe de nada não, o procedimento está errado, não é assim. (E2)

Ter apenas enfermeiros especialistas no CPN é um diferencial positivo para a assistência. (E1)

Nós sempre, desde o início, tivemos uma dificuldadezinha com a parte médica. Ainda há muitos deles que não confiam na enfermeira, então, às vezes, vem, querem tomar uma ação nossa que nós estejamos fazendo, que acha que estamos fazendo errado ou, então discordam daquilo. (E2)

Sobre a divisão do trabalho as participantes fizeram as seguintes narrativas:

O trabalho burocrático, às vezes, dificulta a assistência. Porque partejar não é fácil, precisa coragem, paciência, tempo [...] porque é muito fácil você acelerarum parto. (E5)

Na minha atuação eu sempre procuro dar realmente essa oportunidade de a gestante ter esse parto humanizado, gosto muito do plano de parto, gosto muito de acompanhar as visitas às gestantes, de realmente tirar as dúvidas delas, fazer as visitas, as orientações. (E1)

Parte burocrática, nós fazemos sempre, como em toda instituição, são as papeladas, preenchimento, partograma, anotações. Mas assim, na gestão em si, o que eu acho que eu sempre faço é tentar organizar o setor, no caso, faltou material ou faltou algum técnico, a gente tenta remanejar alguém para que não fique desfalcado, então assim, eu tento ver muito isso. (E3)

Aqui nós temos só uma enfermeira, que o ideal seria duas enfermeiras no CPN. Então nós ficamos com a parte burocrática e a parte assistencial. Então, acumulando muito o trabalho, não é, porque é muita papelada, a burocracia é grande, nós temos que preencher vários papéis. Além do que, tem o livro de admissão de lá, tem o censo, tem os indicadores e o próprio prontuário que a gente preenche, então é muita papelada, é muita burocracia, que a gente faz. (E2)

A questão da verificação do censo, que é onde nós temos o controle da entrada e saída das pacientes, o registro da ocorrência, de qualquer coisa que venha a ocorrer fora do padrão do plantão, e também o registro das avaliações, porque não adianta nós avaliarmos e não registrar. Então, também fica responsável por fazer o registro de todas as avaliações das pacientes. (E6)

Complementaram acerca do Mercado de trabalho, com as seguintes falas:

Fazer a especialização em enfermagem obstétrica foi um acerto pra mim, porque com a rede cegonha abriu muito espaço para quem era especialista. (E2) 
Eu acho que a questão da educação continuada [é importante]. Porque por mais que a equipe já esteja acostumada, já trabalhe na área, eu acho que tem que estar sempre renovando, sempre tratar desse assunto, até mesmo para ficar fresquinha para a equipe toda. (E6)

O CPN foi uma nova oportunidade de trabalho, que agregou o que eu já fazia em centro obstétrico, mas com maiores responsabilidades, porque agora nós somos responsáveis por mais coisas. Eo jeito de fazer mudou, porque a enfermagem deixa a mulher mais livre também. (E5)

Fiz a residência na área de obstetrícia e acho que devo muito a essa oportunidade. Aproveitei quando abriu vaga no CPN e tento fazer aqui tudo o que aprendi naquela época, porque na residência pude atuar partejando. Isso ajudou muito. (E1)

\section{E no contexto do Quadro de Valores explicitaram:}

Vejo como é importante a portaria do Ministério da Saúde que regulamenta o CPN, informando acerca da obrigatoriedade do enfermeiro obstetra no processo e exigindo isso nos serviços. Mas vejo que ainda há um caminho longo a seguir, pois nem tudo da portaria é visto na prática. (E1)

Nosso trabalho é orientado pelas normas do conselho profissional e do Ministério da Saúde, mas infelizmente, ainda falta muita coisa para tudo sair do papel. Talvez, se houvesse mais monitoramento dos processos por quem deve fazer isso, a situação seria diferente. (E5)

OCPN trouxe mais autonomia para a enfermeira, porque nos deixa mais livres para desenvolver nosso trabalho. Ainda há dificuldades que não foram superadas. Posso citar a assinatura da Autorização de Internação Hospitalar que não foi liberada ainda para que a gente faça aqui. Tem regulamentação para isso, mas não tem sido implantado nos CPN. (E3)

\section{DISCUSSÃO}

O primeiro tema Conhecimento e Autonomia envolve os tipos de atividades profissionais, controle e autonomia ocupacional, além dos tipos de conhecimentos e competências. ${ }^{14}$ Nessa perspectiva, salienta-se que para que o enfermeiro tenha autonomia no CPN é imprescindível amplo conhecimento dos cuidados à parturiente e puérpera e destreza na prática do cuidado.

As ações da enfermeira obstétrica no CPN fortificam a valorização da assistência humanizada, pois estes profissionais colaboram com a promoção da saúde da mulher e do recémnascido proveniente de um parto vaginal. Isso facilita o processo de trabalho de parto e resulta em diversos benefícios para ambos. ${ }^{7}$
Nesse âmbito, o enfermeiro possui um papel importante no cenário obstétrico, especialmente como potencializador de práticas saudáveis para o parto e nascimento, embasadas em evidências científicas. ${ }^{2}$ Para isso, o profissional precisa de constante aprimoramento, pois somente a partir do conhecimento é possivel a obtenção do respeito social e consequente autonomia para o desenvolvimento das atividades laborais. ${ }^{14}$

Vale ressaltar que, diante de sua formação ético-humanística, o enfermeiro possui uma conduta de acolhimento com uma assistência baseada não apenas em técnicas, mas também em práticas relacionadas com diálogos entre profissionais e usuários. Isso denota um cuidado singular integrado por qualidades como afeto, respeito e segurança para com as mulheres, valorizando seu protagonismo no parto. ${ }^{7}$

Salienta-se a importância das construções teórico-reflexivas oportunizadas durante o processo formativo em obstetrícia para enfermeiros, pois isso possibilita transformação de pensamentos e práticas que contribuem para a qualificação dos serviços prestados no sistema de saúde. ${ }^{17}$

Por outro lado, deve-se atentar para a necessidade de contínuo aprimoramento dos conhecimentos dos enfermeiros, visto que novas práticas são implementadas constantemente na enfermagem obstétrica. Isso exige dedicação à atualização profissional em um ambiente que apresenta variadas dificuldades no cotidiano. ${ }^{18}$

A atuação do enfermeiro no CPN permite a aplicação dos conhecimentos apreendidos ao longo do seu processo de formação, o que pode resultar em ampliação de sua autonomia no desenvolvimento das atividades. Porém, também requer dos profissionais maior esforço para abarcar as diversas responsabilidades resultantes deste processo.

O tema Credencialismo visa identificar o grau de abertura e fechamento das credenciais e requisitos de acesso à formação, evolução e comparação. ${ }^{14}$ Os enfermeiros, principalmente especialistas em enfermagem obstétrica, lutam pela conquista de autonomia e reconhecimento de suas competências na assistência ao trabalho de parto e parto, porém, é necessário que estejam cientes de suas responsabilidades e que assumam os resultados dos seus cuidados da mesma maneira que os profissionais médicos; devendo ser imprescindível a discussão sobre esta questão, estabelecendo os cuidados preventivos e as consequências legais. ${ }^{19}$

Ressalta-se que a Lei do Exercício Profissional de Enfermagem, aprovada no ano de 1986, confirma a atuação do enfermeiro obstétrico no cuidado integral no trabalho de parto/parto, ressaltando a sua autonomia e competência para o oferecimento de cuidado ao processo de parturição. ${ }^{20}$

Além disso, para melhor reconhecimento social desta categoria profissional, é necessário agregar valor ao cuidado durante $o$ ato de partejar, sem esquecer as questões de gênero que envolvem o processo. Geralmente, são mulheres enfermeiras cuidando de outras mulheres em parturição, em um ambiente de antigo domínio masculino. ${ }^{21}$ 
Também é necessária a construção de relações de confiança entre os profissionais, com a utilização de comunicações claras e respeitosas, bem como o desenvolvimento de equipes que promovam a colaboração interprofissional. ${ }^{22}$ No entanto, a narrativa evidencia o frágil vínculo entre a equipe como uma dificuldade para a implementação das práticas recomendadas pela OMS, pois, ainda existe a desvalorização das competências do enfermeiro por parte da equipe médica; necessitando a maior disseminação da importância do cuidado humanizado.

Essa relação interprofissional se coloca como um desafio a ser superado pelas enfermeiras para a qualificação do trabalho desenvolvido no CPN. Também emerge como possibilidade de ampliação da importância da categoria profissional para o desenvolvimento de atividades no âmbito da obstetrícia.

Nesse sentido, o desenvolvimento da assistência em saúde materno-infantil por profissionais qualificados para atuação em equipe, surge como importante desafio em diversos países. As experiências positivas de colaboração devem ser potencializadas como norte para os sistemas de saúde replicarem. ${ }^{23}$

$\mathrm{O}$ agir coletivo, a partir das normativas vigentes, potencializa o reconhecimento social de uma profissão em determinada atividade, especialmente naquelas realizadas em parte por outra categoria. ${ }^{14}$ Destarte, os enfermeiros dos CPN devem entender a importância da sua formação e das normativas que regem sua atuação profissional, pois isso os credencia para desenvolver as atividades de qualificação do parto e nascimento neste equipamento de saúde.

O tema Divisão do Trabalho envolve posição na classificação socioprofissional oficial, a evolução e comparação no âmbito do trabalho. ${ }^{14}$ Como foi apresentado no eixo anterior é possível perceber o espaço de disputa entre enfermeiros obstetras e médicos.

O enfermeiro possui como atribuições, tarefas diretamente relacionadas à assistência e outras, que envolvem liderança da equipe de enfermagem e o gerenciamento de recursos, sejam físicos, materiais, humanos, financeiros, políticos, sejam de informações. ${ }^{24} \mathrm{O}$ acúmulo de atividades de uma categoria profissional pode ser visto de forma negativa pelas exigências que isso acarreta. No entanto, também pode ser visualizada como uma oportunidade de ampliação do domínio profissional desta categoria em uma área específica de atuação. ${ }^{14}$

Além da assistência voltada aos cuidados, o enfermeiro é responsável pela gestão administrativa do CPN, possuindo inúmeras responsabilidades associadas à parte burocrática, tornando seu trabalho extenso.

Como gerente da assistência de enfermagem, este profissional necessita de conhecimentos, habilidades e atitudes para exercer seu trabalho com eficiência. ${ }^{24}$ As participantes relatam as práticas que consideram parte da gestão: o preenchimento de papeladas, partograma, anotações, registro de ocorrência, admissão em prontuários e organização do setor como ações administrativas. Além disso, citam a questão de dimensionamento de pessoal de enfermagem, delegação das atividades e checagem dos materiais entre suas atribuições de gestão.
$\mathrm{Na}$ área assistencial, porém, as participantes relatam a importância de seu trabalho para a mulher, recém-nascido e familiares no processo de acompanhamento ao parto. Cuidados estes que envolvem todas as etapas nesta situação e devem ser realizados com paciência e organização.

As atribuições gerenciais dos enfermeiros são indispensáveis para os serviços de saúde no mundo, devendo haver um consenso sobre essas competências de gestão e estímulo para despertar discussões acerca do tema, a fim de que o debate promova o conhecimento e definição das ações administrativas do enfermeiro e, principalmente, desperte o desenvolvimento gerencial nestes profissionais. ${ }^{24}$

No entanto, é preciso destacar que os sistemas de saúde devem priorizar o cuidado obstétrico por profissionais capacitados, em detrimento de áreas meramente administrativas. Conciliar as duas questões pode ampliar sobremaneira a qualidade dos serviços oferecidos a população. ${ }^{25}$

As enfermeiras obstétricas possuem atribuições importantes não só nas práticas assistenciais quanto na questão administrativa, sendo essa priorizada por instituições de saúde. Na gestão, os enfermeiros são reconhecidos pela supervisão de pessoal de enfermagem e provimento de recursos necessários para a unidade, tornando a assistência prestada efetiva, porém, essa atribuição requer maior demanda de tempo e dedicação para o serviço, ocasionando, muitas vezes, o distanciamento do enfermeiro das práticas humanizadas. ${ }^{26}$

Essa realidade, ao que parece, é um dos desafios que necessita discussão para seu enfrentamento. As práticas assistenciais e administrativas estão concatenadas, porém na divisão do trabalho, as atividades de gestão consomem um tempo importante que poderia ser disponibilizado para o cuidado direto a parturiente. Torna-se necessária a busca pela harmonia nestas ações para otimização da atuação do enfermeiro no CPN.

No tema Mercado de Trabalho é apresentado o contexto da evolução e caracterização dos requisitos de acesso ao mercado de trabalho e tipologias de carreira ou outros mecanismos de desenvolvimento profissional. ${ }^{14}$

Ressalta-se que uma formação adequada para o processo da assistência obstétrica pode ampliar a qualidade do serviço e a satisfação das usuárias. A prática dos cuidados centrados nas parturientes, porém com entendimento acerca das fragilidades e potencialidades profissionais é responsável por significativas melhoras na atenção. O desenvolvimento das práticas na obstetrícia deve ser construído a partir de trabalhos formativos que enfatizem as demandas dos envolvidos na situação. ${ }^{27,28}$

Ademais, a formação em obstetrícia para a enfermagem deve utilizar ferramentas embasadas em conceitos teóricos discutidos constantemente. A partir disso, as práticas cotidianas tendem a ser realizadas com maior precisão técnica, o que agrega confiança e segurança ao profissional e à mulher durante a assistência prestada. ${ }^{29,30}$

Além disso, a formação específica em obstetrícia é apresentada nas narrativas das participantes como essencial para qualificação das atividades desenvolvidas no CPN. Esse 
fator o delimita como um espaço conquistado por aqueles que possuem credenciais em um nicho da enfermagem: a obstetrícia.

Nessa perspectiva, a identidade profissional da enfermagem obstétrica é fortalecida na sua agregação ao CPN, pois a ligação com esta instituição pública permite aos enfermeiros a ampliação de sua visibilidade social, sendo um dos benefícios ocasionados por esta situação. Em contraponto com essa questão, devido à aproximação entre categoria e instituição, é necessário que as ações desenvolvidas no CPN produzam desfechos positivos, caso contrário, a enfermagem obstétrica poderá ser responsabilizada pelas possíveis fragilidades encontradas no cotidiano dos serviços.

Ressalta-se que o CPN como ponto de atenção recentemente implementado no SUS, oportunizou a abertura de um amplo campo de atuação para a enfermagem brasileira. Diferentemente do centro obstétrico, o CPN possui o enfermeiro como responsável pela linha de cuidado materno-infantil, ampliando a importância social deste profissional. ${ }^{31}$ Conforme Rodrigues,${ }^{14}$ isso pode denotar a demarcação de espaço profissional alcançado por uma categoria, por influência no Estado, que induz ampliação do mercado de trabalho neste conjunto de indivíduos.

No último tema que trata do Quadro de Valores são apresentadas percepções dos membros da profissão e instituições representativas sobre a autonomia e o controle ocupacional, as condições de trabalho e emprego, os critérios de avalição da qualidade dos serviços, das competências dos membros da profissão, da defesa do interesse público, da responsabilidade e do altruísmo. ${ }^{14}$

As entrevistadas ressaltam, em seus depoimentos, a importância da regulamentação do CPN para a enfermagem obstétrica, pois isso delimita o campo de atuação, com organização das atividades desenvolvidas no cotidiano. No entanto, há fragilidade relatada no monitoramento das atividades deste serviço de saúde, o que dificulta a implementação das ações descritas nas normas que o regem.

O monitoramento e avaliação no SUS são essenciais para a qualificação do atendimento ofertado à população. $\mathrm{E}$ a enfermagem sempre desempenhou importante papel para que isso ocorresse no país, propiciando a melhora contínua do sistema. ${ }^{11}$ Quando isso não ocorre, produz limitações para as ações do enfermeiro, como descrito pelas participantes.

A normatização, sendo utilizada como base para as ações implementadas no CPN, é capaz de resguardar o potencial das enfermeiras obstétricas para as atividades de gestão e cuidado materno-infantil. Ao contrário, quando as normas não são consideradas, há possibilidade de prejudicar o desenvolvimento do trabalho que legalmente o enfermeiro pode desempenhar. A negação da assinatura pelas enfermeiras da Autorização de Internação Hospitalar é um exemplo citado pelas participantes.

Essa ação encontra embasamento nas normativas do Ministério da Saúde ${ }^{9}$ e talvez não seja praticada devido às disputas com outras categorias profissionais, que não permitem a ocupação deste espaço pelas enfermeiras obstétricas. Isso limita sua atuação no $\mathrm{CPN}$, pois não permite que sejam responsáveis oficialmente pelo internamento, acompanhamento e alta hospitalar da mulher e do recém-nascido, conforme determina o regramento do SUS.

O reconhecimento das atividades inerentes aos integrantes do grupo ocupacional responsável pelo trabalho é essencial para a construção dos aspectos profissionais que fomentam o contínuo desenvolvimento das ações daquele coletivo. Conhecer e reconhecer suas limitações e potencialidades possibilita a ampliação do interesse social e visibilidade da profissão. ${ }^{14}$

\section{CONCLUSÕES E IMPLICAÇÕES PARA A PRÁTICA}

O Centro de Parto Normal não é campo de domínio exclusivo de um profissional, e emerge como cenário de discussões para melhora do cuidado materno-infantil, bem como dá maior visibilidade à enfermagem obstétrica no sistema público de saúde. Além disso, denota a influência desta categoria junto ao Estado para ampliação do mercado de trabalho e inserção da enfermeira obstétrica no cenário do parto, algo que deve ser melhor explorado.

Este ponto de atenção é relevante por ampliar a área de atuação da enfermagem obstétrica e garantir por meio de normativas a autonomia laboral destes profissionais. No entanto, em desrespeito às normas vigentes essa autonomia ainda não é total, ocasionada, possivelmente, por disputas com outras categorias, pois observa-se a necessidade de maior reconhecimento das competências do enfermeiro por parte de outros profissionais.

Salienta-se que a formação específica na área, com valorização das evidências científicas, amplia as potencialidades de qualificação do cuidado prestado. Também, a concatenação das ações de assistência e gestão na área materno-infantil podem ser utilizadas para se obter maior reconhecimento social da enfermeira obstétrica. Porém, o trabalho burocrático exacerbado em detrimento do cuidado direto pode limitar esse processo.

Salienta-se como limitação do estudo, a realização em um equipamento com especificidades contextuais. Porém, como integrante da rede de atenção materno-infantil, seguem diretrizes elencadas pela gestão do sistema de saúde, apresentando, portanto, uma realidade que pode ser similar às encontradas em outros cenários.

Ainda que as políticas públicas estejam apoiando a inserção das enfermeiras obstétricas na assistência ao parto e nascimento, especialmente nos CPN, a transformação do modelo assistencial continua sendo considerada um desafio que demanda esforços da gestão, dos profissionais de saúde e da sociedade.

\section{FINANCIAMENTO}

Conselho Nacional de Desenvolvimento Científico e Tecnológico. Concessão de bolsa de Iniciação Científica PIBIC/ CNPq a Ana Carolina de Melo Farias Teixeira, no ano de 2018. 


\section{CONTRIBUIÇÕES DOS AUTORES}

\author{
Concepção do desenho do estudo: Antonio Rodrigues \\ Ferreira Júnior. \\ Coleta de dados: Antonio Rodrigues Ferreira Júnior. Ana
} Carolina de Melo Farias Teixeira.

Análise dos dados: Antonio Rodrigues Ferreira Júnior. Luciana Camila dos Santos Brandão. Ana Carolina de Melo

Farias Teixeira. Alexandrina Maria Ramos Cardoso.

Interpretação dos dados: Antonio Rodrigues Ferreira Júnior. Luciana Camila dos Santos Brandão. Alexandrina Maria Ramos Cardoso.

Redação e revisão crítica do manuscrito Antonio Rodrigues Ferreira Júnior. Luciana Camila dos Santos Brandão. Alexandrina Maria Ramos Cardoso.

Aprovação da versão final: Antonio Rodrigues Ferreira Júnior. Luciana Camila dos Santos Brandão. Ana Carolina de Melo Farias Teixeira. Alexandrina Maria Ramos Cardoso.

Responsabilidade pelo conteúdo intelectual, acurácia e integridade de quaisquer partes do artigo:

Antonio Rodrigues Ferreira Júnior. Luciana Camila dos Santos Brandão. Ana Carolina de Melo Farias Teixeira. Alexandrina Maria Ramos Cardoso.

\section{EDITOR ASSOCIADO}

\section{Ana Luiza de Oliveira Carvalho}

\section{REFERÊNCIAS}

1. Bittencourt DAS, Domingues RM, Reis LG, Ramos MM, Leal MD Adequacy of public maternal care services in Brazil. Reproductive Health 2016 out; 13 (13 Suppl. 3):120. http://dx.doi.org/10118/s12978016-0229-6.

2. World Health Organization. WHO recommendations: intrapartum care for a positive childbirth experience. Geneva: WHO; 2018.

3. United Nations Population Fund, International Confederation of Midwives, World Health Organization. The state of the world's midwifery 2014: a universal pathway - a woman's right to health. New York: UNPF; 2014.

4. Dias MAB, Domingues RM, Schilithz AO, Nakamura-Pereira M, Leal MC Factors associated with cesarean delivery during labor in primiparous women assisted in the Brazilian Public Health System: data from a national survey. Reproductive Health 2016 out; 13(Suppl. 3):114. http:// dx.doi.org/10118/s12978-016-0231-z.

5. Esteves-Pereira AP, Deneux-Tharaux C, Nakamura-Pereira M, Saucedo $\mathrm{M}$, Bouvier-Colle MH, Leal MC. Caesarean delivery and postpartum maternal mortality: A population-based case control study in Brazil. PLoS One. 2016 abr;11(4):E0153396. http://dx.doi.org/10.1371/journal. pone.0153396. PMid:27073870.

6. Ye J, Betrán AP, Guerrero Vela M, Souza JP, Zhang J. Searching for the optimal rate of medically necessary cesarean delivery. Birth. 2014 set;41(3):237-44. http://dx.doi.org/10.1111/birt.12104. PMid:24720614.

7. Silva FMB, Paixão TCR, Oliveira SMJV, Leite JS, Riesco MLG, Osava $\mathrm{RH}$. Assistência em um centro de parto segundo as recomendações da Organização Mundial de Saúde. Rev Esc Enferm USP. 2013 out;47(5):1031-8. http://dx.doi.org/10.1590/S0080-623420130000500004. PMid:24346440.

8. Portaria no 1.459 de 24 de junho de 2011 (BR). Institui, no âmbito do Sistema Único de Saúde - SUS - A Rede Cegonha. Diário Oficial da União, Brasília (DF), 25 jun 2011.

9. Portaria no 904 de 29 de maio de 2013 (BR). Estabelece diretrizes para implantação e habilitação de Centro de Parto Normal (CPN), no âmbito do Sistema Único de Saúde (SUS), para o atendimento à mulher e ao recém-nascido no momento do parto e do nascimento, em conformidade com o Componente PARTO E NASCIMENTO da Rede Cegonha, e dispõe sobre os respectivos incentivos financeiros de investimento, custeio e custeio mensal. Diário Oficial da União, Brasília (DF), 30 mai 2013.

10. Andrade LO, Félix ESP, Souza FS, Gomes LOS, Boery RNSO. Práticas dos profissionais de enfermagem diante do parto humanizado. Rev enferm UFPE on line 2017; jun;11(6):2576-85. http://dx.doi.org/10.5205/ reuol.9799-86079-1-RV.1106sup201712.

11. Silva MCN, Machado MH. Sistema de Saúde e Trabalho: desafios para a Enfermagem no Brasil. Ciênc. Saúde Coletiva. 2020 jan;25(1):7-13. http://dx.doi.org/10.1590/1413-81232020251.27572019.

12. Ferreira Júnior AR, Fontenele MEP, Albuquerque RAS, Gomes FMA, Rodrigues MENG. A socialização profissional no percurso de técnico a enfermeiro. Trab Educ Saúde. 2018 set/dez 16(3):1321-35. http:// dx.doi.org/10.1590/1981-7746-sol00152.

13. Minayo MCS. O Desafio do Conhecimento: pesquisa qualitativa em saúde. 14르 ed. São Paulo: HUCITEC; 2014.

14. Rodrigues ML. Profissões: lições e ensaios. 1a ed. Coimbra: Grupo Almeida; 2012.

15. Tong A, Sainsbury P, Craig J. Consolidated criteria for reporting qualitative research (COREQ): a 32 -item checklist for interviews and focus groups. Int J Qual Health Care. 2007;19(6):349-57. http://dx.doi.org/10.1093/ intqhc/mzm042. PMid:17872937.

16. Resolução $n^{\circ} 466 / 2012$ (MS). Dispõe sobre pesquisa envolvendo seres humanos. Conselho Nacional de Saúde, Brasília (DF); 2012.

17. Lima MFG, Pequeno AMC, Rodrigues DP, Carneiro C, Morais APP, Negreiros FDS. Developing skills learning in obstetric nursing: approaches between theory and practice. Rev Bras Enferm. 2017 set/out;70(5):105460. http://dx.doi.org/10.1590/0034-7167-2016-0665. PMid:28977234.

18. Gama SG, Viellas EF, Torres JA, Bastos MH, Brüggemann OM, Theme Filha MM et al. Labor and birth care by nurse with midwifery skills in Brazil. Reprod Health. 2016 out;13(S3, Suppl 3):123. http://dx.doi. org/10.1186/s12978-016-0236-7. PMid:27766971.

19. Nunes JH, Silva MFGD. O trabalho de enfermeiras(os) obstetras na assistência a nascimentos e partos: profissionalização e tensões identitárias. Revista da ABET. 2016;15(2):165-81.

20. Medeiros RMK, Teixeira RC, Nicolini AB, Alvares AS, Corrêa ACP, Martins DP. Cuidados humanizados: a inserção de enfermeiras obstétricas em um hospital de ensino. Rev Bras Enferm. 2016 nov/dez;69(6):1091-8. http://dx.doi.org/10.1590/0034-7167-2016-0295. PMid:27925085.

21. Costa RLM, Costa ILS. Um ponto de Resistência: enfermagem, medicina e gênero no contexto hospitalar. Cad Espaço Feminino. 2017;30(2):35766. http://dx.doi.org/10.14393/CEF-v30n2-2017-20.

22. Araújo CAS, Figueiredo KF. Brazilian nursing professionals: leadership to generate positive attitudes and behaviours. Leadersh Health Serv (Bradf Engl). 2019 jan;32(1):18-36. http://dx.doi.org/10.1108/LHS-032017-0016. PMid:30702040.

23. Hobbs AJ, Moller AB, Kachikis A, Carvajal-Aguirre L, Say L, Chou D. Scoping review to identify and map the health personnel considered skilled birth attendants in low-and-middle income countries from 20002015. PLoS One. 2019 fev; 14(2):e0211576. http://dx.doi.org/10.1371/ journal.pone.0211576. PMid:30707736.

24. Nelson-Brantley HV, David Bailey K, Batcheller J, Bernard N, Caramanica L, Snow F. Grassroots to Global: The future of nursing leadership. J Nurs Adm. 2019 mar;49(3):118-20. http://dx.doi.org/10.1097/ NNA.0000000000000723. PMid:30789554.

25. Burrowes S, Holcombe SJ, Jara D, Carter D, Smith K. Midwives' and patients' perspectives on disrespect and abuse during labor and delivery care in Ethiopia: a qualitative study. BMC Pregnancy Childbirth 2017 ago;17(1):263. http://dx.doi.org/10.1186/s12884-017-1442-1. PMid:28830383.

26. Almeida OSC, Gama ER, Bahiana PM. Humanização do parto - A atuação dos enfermeiros. Rev Enfermagem Contemporânea. 2015;4(1):79-90. http://dx.doi.org/10.17267/2317-3378rec.v4i1.456.

27. Perdok H, Jans S, Verhoeven C, Henneman L, Wiegers T, Mol BW et al Opinions of maternity care professionals and other stakeholders about 
integration of maternity care: a qualitative study in the Netherlands. BMC Pregnancy Childbirth. 2016 jul;16(1):188. http://dx.doi.org/10.1186/ s12884-016-0975-z. PMid:27459967.

28. Ekström AC, Thorstensson S. Nurses and midwives professional support increases with improved attitudes - design and effects of a longitudinal randomized controlled process-oriented intervention. BMC Pregnancy Childbirth. 2015 out;15(1):275. http://dx.doi.org/10.1186/s12884-0150712-z. PMid:26503218.

29. Gray B, Cavner J. Using Simulation to Enhance Education in an Obstetrical Nursing Course. Nurs Educ Perspect. 2017;38(1):40-
1. http://dx.doi.org/10.1097/01.NEP.0000000000000099. PMid:29194244.

30. Hogewood C, Smith T, Etheridge S, Britt S. Clinical Boot Camp: An innovative simulation experience to prepare nursing students for obstetric and pediatric clinicals. Nurs Educ Perspect. 2015 nov/dez;36(6):410-1. http://dx.doi.org/10.5480/15-1677. PMid:26753307.

31. Soares YKC, Melo SSS, Guimarães TMM, Feitosa VC, Gouveia MTO Satisfação das puérperas atendidas em um Centro de Parto Normal. J Nurs UFPE on line 2017 nov; 11(Suppl. 11):4563-73. http://dx.doi. org/10.5205/reuol.11138-99362-1-SM.1111sup201704 